\title{
Right pneumonectomy for invasive pulmonary mucormycosis
}

\author{
Natalie Van Ochten, BS, ${ }^{\mathrm{a}}$ Madhuri V. Rao, MD, ${ }^{\mathrm{b}}$ Amit Bhargava, MD, ${ }^{\mathrm{b}}$ Rafael S. Andrade, MD, MHA, ${ }^{\mathrm{b}}$ and \\ Ilitch Diaz-Gutierrez, MD, ${ }^{b}$ Minneapolis, Minn
}

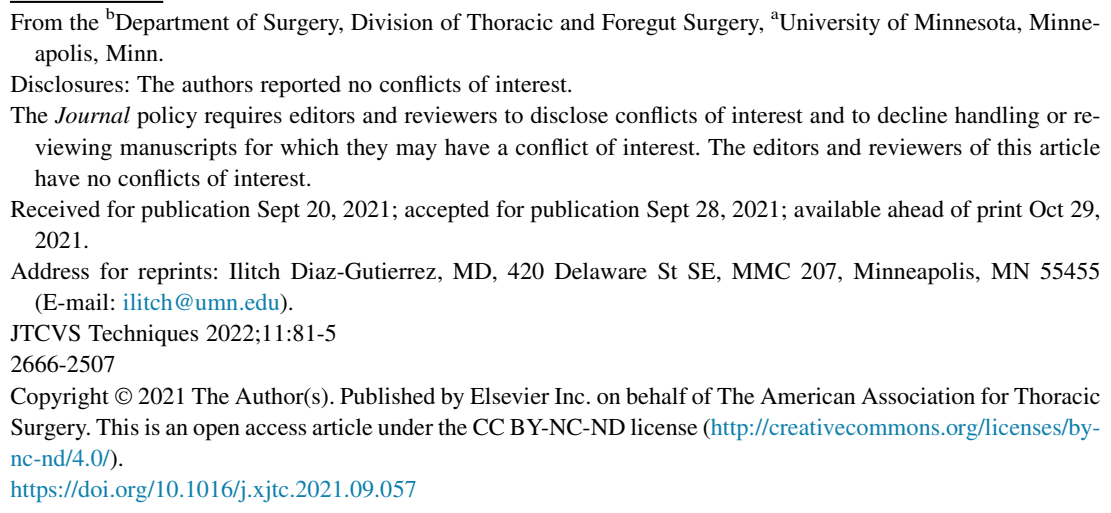

Video clip is available online.

Mucormycosis is a rare but potentially lethal opportunistic infection that affects immunocompromised individuals. Invasive pulmonary mucormycosis comprises $24 \%$ of the different clinical forms of the disease. ${ }^{1}$ Mortality ranges from $44 \%$ to $76 \%$, secondary to rapid angioinvasion and dissemination to nearby organs. ${ }^{1-3}$ Early surgical debridement of all diseased tissue, in combination with correction of immunosuppression and antifungal therapy, are paramount in optimizing the chance for survival. In certain situations, aggressive surgical debridement requires a pneumonectomy however, this has been rarely described in the literature. ${ }^{4,5}$ We present a challenging case of invasive pulmonary mucormycosis affecting the right hilum, managed with a median sternotomy first, to control the great vessels, followed by a posterolateral thoracotomy and pneumonectomy (Video 1).

\section{CASE}

An institutional review board exemption was obtained for this retrospective review and the patient provided informed consent. A 21-year-old woman presented in diabetic ketoacidosis with dry cough, night sweats, and respiratory failure. Evaluation was remarkable for leukocytosis of $10 \times 10^{9} / \mathrm{L}$, elevated procalcitonin of $21 \mathrm{ng} / \mathrm{mL}$, and a right hilar inflammatory mass on computed tomography scan (Figure E1). Infectious evaluation including blood and

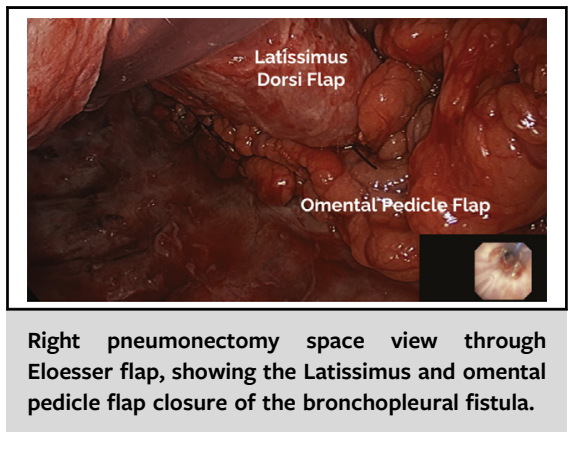

\begin{abstract}
CENTRAL MESSAGE
Hilar pulmonary mucormycosis is life-threatening and mandates aggressive surgical resection. A young patient merits nearly any salvage intervention to manage complications.
\end{abstract}

See Commentaries on pages 86 and 87.

bronchial cultures, HIV, tuberculosis, legionella, beta-D glucan, and galactomannan were negative. Transbronchial biopsies showed broad-based hyphae invading tissue, suggestive of zygomycosis. Treatment was started with intravenous amphotericin B $50 \mathrm{mg} / \mathrm{d}$ however, she showed progression of disease after 10 days of therapy. We discussed her in multidisciplinary conference and decided to proceed urgently with a right pneumonectomy, because the inflammatory mass appeared to encase the right main pulmonary artery and the main stem bronchus.

First, we performed a femoral cutdown, cannulated the femoral vessels, and connected to the cardiopulmonary bypass circuit in preparation for emergent bypass in case of rupture of the right main pulmonary artery, yet this was not required. Second, we performed a sternotomy and successfully dissected the inflamed right main pulmonary artery. We then divided the right main pulmonary artery and right pulmonary veins with a vascular stapler (Figure E2). We closed the chest and repositioned for a right posterolateral thoracotomy. Third, we continued with an extrapleural dissection because of the extensive inflammatory process in the posterior mediastinum (Figure E3). We separated the esophagus from the carina with some difficulty and confirmed absence of esophageal injury with endoscopy. 


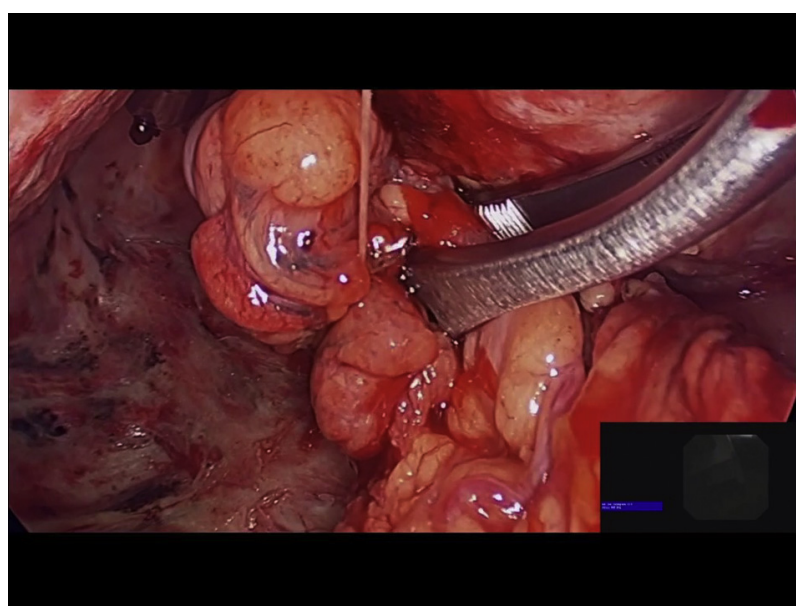

VIDEO 1. Right extrapleural pneumonectomy for invasive pulmonary mucormycosis. First, we performed a femoral cut-down and cannulated peripherally in preparation for emergent cardiopulmonary bypass (not required). Second, we performed a median sternotomy and divided the right main pulmonary artery and right pulmonary veins. Third, we closed the patient and repositioned for a right posterolateral thoracotomy. Next, we dissected in an extrapleural plane because of the extensive inflammatory process. We separated the esophagus and the carina with some difficulty, performed a carinal plasty to exclude the damaged tissue from the staple line, and reinforced it with a Latissimus flap. Postoperatively, the patient had dehiscence of the bronchoplasty, empyema, and respiratory failure. This was managed with an extra-long tracheostomy with the balloon placed distal to dehiscence, in the mid-left mainstem bronchus, and an Eloesser flap to control the pneumonectomy space. A few days later, the bronchopleural fistula was finally closed with an omental pedicle flap. The patient ultimately recovered and her Eloesser flap was closed on postoperative day 143. Video available at: https://www.jtcvs.org/article/S26662507(21)00739-2/fulltext.

The membranous portion of the carina appeared damaged and friable; therefore, we decided to perform a carinal plasty to exclude the damaged tissue. For the carinal plasty we used interrupted 4-0 vicryl dyed suture (Figure 1). The proximal part of the left main stem bronchus was mobilized bluntly from the carina, to release tension. We used a Latissimus flap to reinforce the carinal plasty, which in our experience is a robust muscle that can achieve excellent coverage.

On postoperative day 10 she had dehiscence of the bronchoplasty, empyema, and respiratory failure. She was managed with an extra-long tracheostomy with the balloon placed distal to dehiscence in the mid-left mainstem bronchus, an Eloesser flap to control the pneumonectomy space, and a feeding tube to optimize nutrition. On postoperative day 14, the bronchopleural fistula was covered with an omental pedicle flap through the open-window thoracostomy (Figure 2). We did not attempt to close the carina dehiscence because of the poor tissue quality and severe inflammation. We avoided a transpericardial approach as described by Perelman and colleagues, ${ }^{6}$ because this would entail reopening the sternotomy and we believed this approach to be too risky for our patient. She ultimately recovered, discharged to a rehabilitation facility on postoperative day 67, and her Eloesser flap was closed on postoperative day 143 . She is receiving long-term posaconazole and has no evidence of recurrence at 12 months (Figure E4). Final pathology was consistent with invasive mucormycosis, necrotizing granulomatous inflammation, tissue necrosis, abscess, and surrounding acute lung injury (Figure E5). Margins were uninvolved by fungal invasion.

An airway dehiscence with bronchopleural fistula was a likely occurrence in this particular case. In retrospect, we could have performed a right thoracostomy at the time of the index operation with the intent to turn an expected bronchopleural fistula into a more manageable problem. At the time of the pneumonectomy with carinal plasty, we did not perceive any undue tension; however further tracheal mobilization could have been undertaken. Had we used cardiopulmonary bypass, we could have divided the left inferior pulmonary ligament, performed a pericardial hilar release, and mobilized the distal left main stem bronchus to further minimize tension.
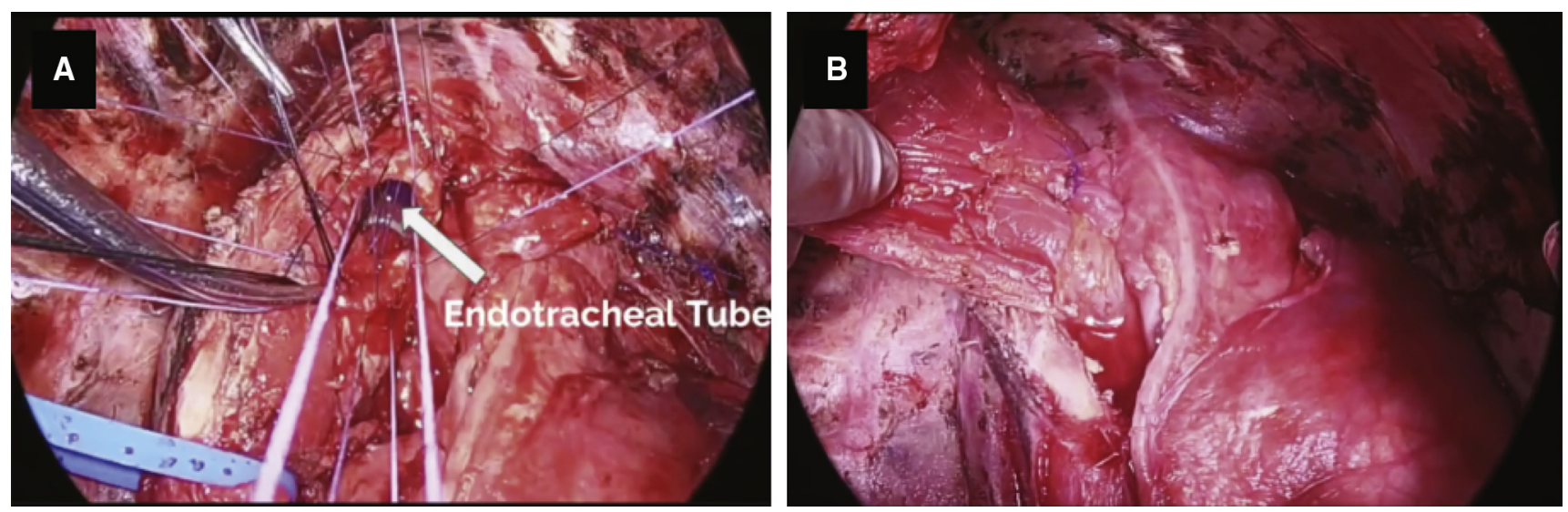

FIGURE 1. Carinal plasty (A), and reinforcement with latissimus dorsi flap (B). 

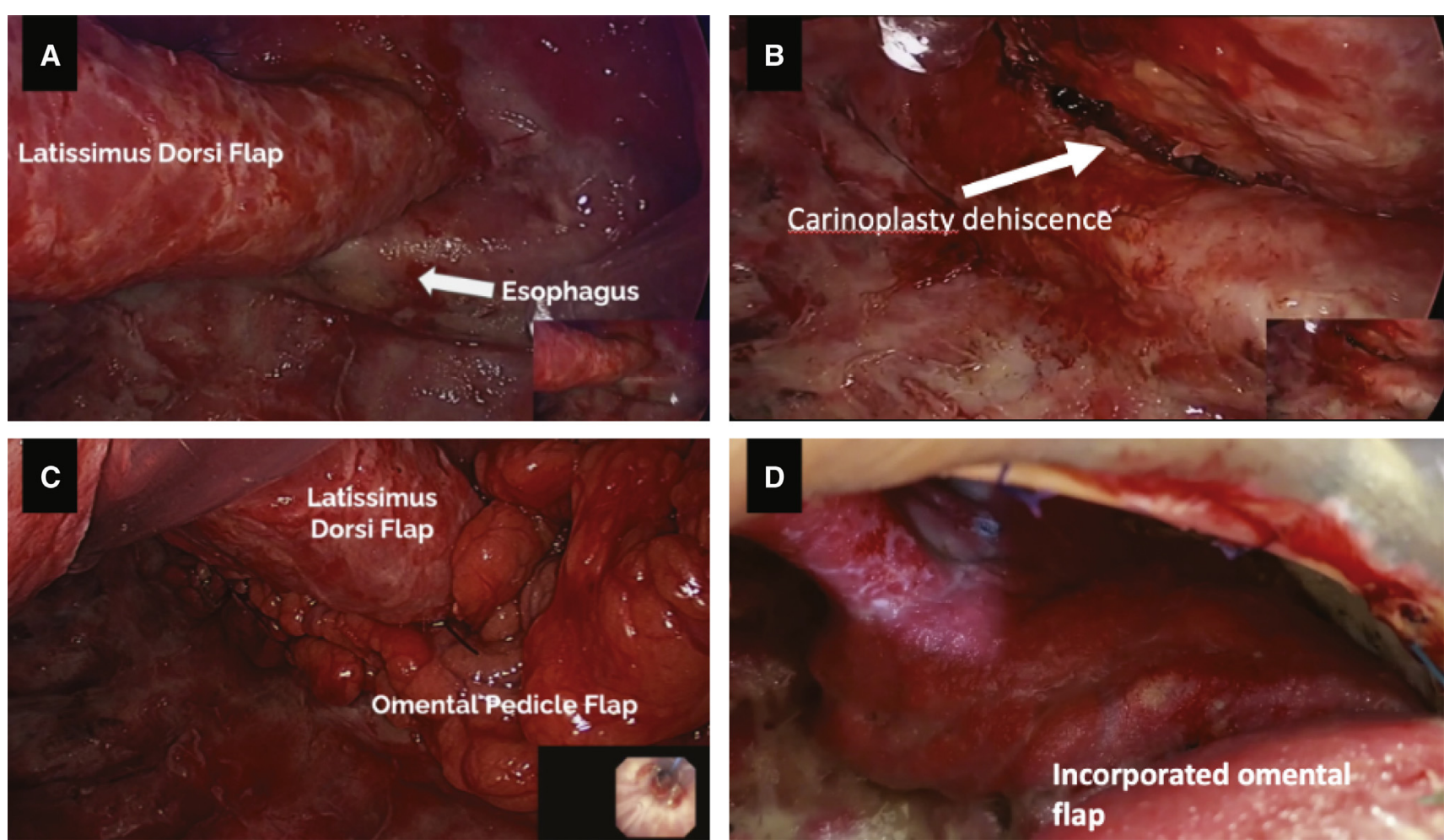

FIGURE 2. Right pneumonectomy space view through the Eloesser flap, showing Latissimus flap (A), site of carinal dehiscence (B), omental pedicle flap (C), and incorporated omental flap (D).

\section{CONCLUSIONS}

Hilar pulmonary mucormycosis is life-threatening and mandates aggressive surgical resection. A young patient merits nearly any salvage intervention to manage complications.

\section{References}

1. Petrikkos G, Skiada A, Lortholary O, Roilides E, Walsh TJ, Kontoyiannis DP. Epidemiology and clinical manifestations of mucormycosis. Clin Infect Dis. 2012;54(Suppl 1):S23-34.
2. Torres-Narbona M, Guinea J, Martínez-Alarcón J, Muñoz P, Gadea I. Emilio Bouza as a representative of the MYCOMED Zygomycosis Study Group. Impact of zygomycosis on microbiology workload: a survey study in Spain. J Clin Microbiol. 2007;45:2051.

3. Connor BA, Anderson RJ, Smith JW. Mucor mediastinitis. Chest. 1979;75:524-6.

4. Majid AA, Yii NW. Granulomatous pulmonary zygomycosis in a patient without underlying illness: computed tomographic appearances and treatment by pneumonectomy. Chest. 1991;100:560-1.

5. Vercillo MS, Liptay MJ, Seder CW. Early pneumonectomy for pulmonary mucormycosis. Ann Thorac Surg. 2015;99:e67-8.

6. Perelman MI, Rymko LP, Ambatiello GP. Bronchopleural fistula: surgery after pneumonectomy. In: Grillo H, Eschapasse H, eds. International Trends in Thoracic Surgery. 2. WB Saunders; 1987. 

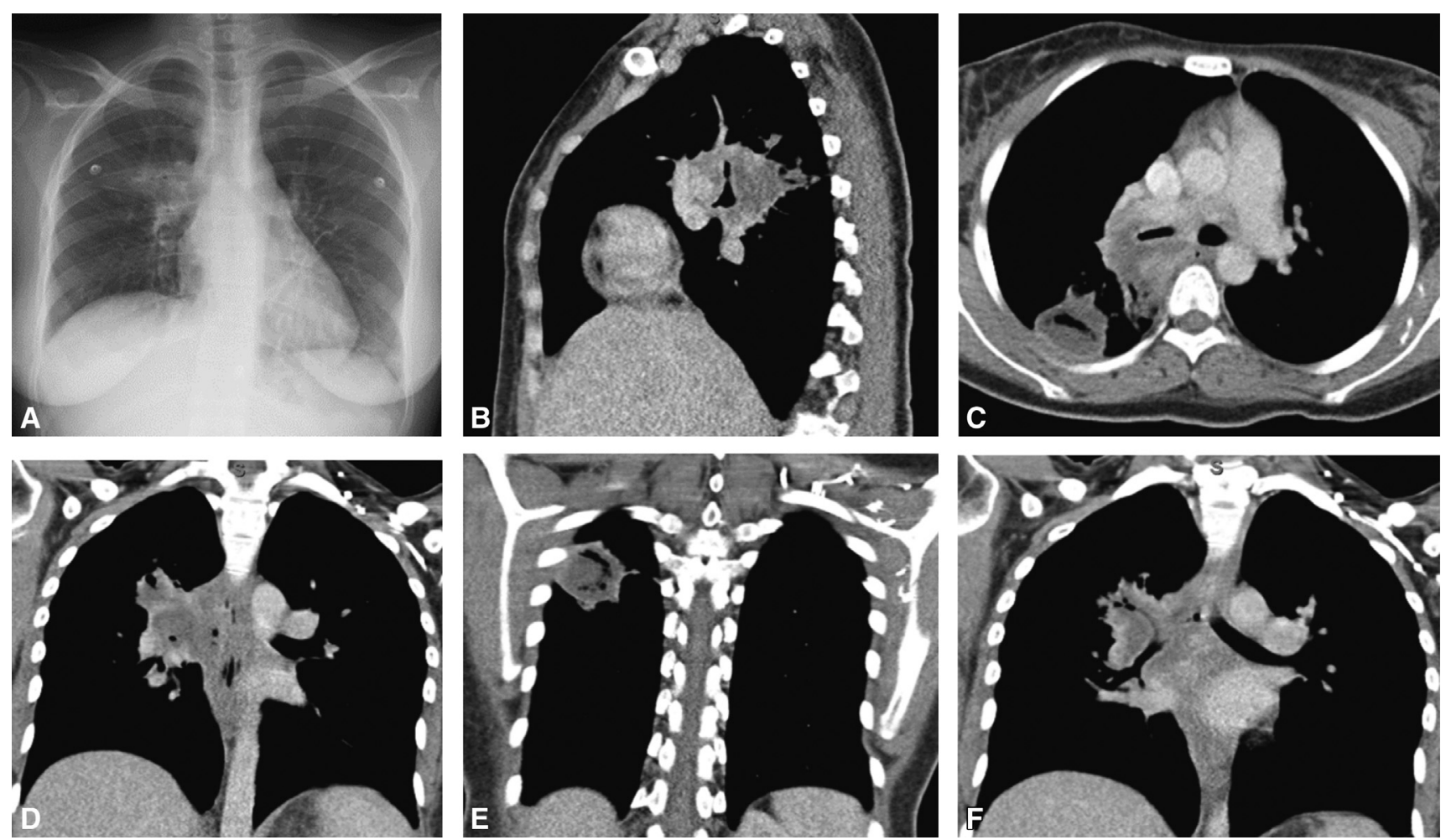

FIGURE E1. Chest radiograph (A) and computed tomography scan (B-F) showing an inflammatory mass extending into the right hilum, encasing the right mainstem bronchus, and behind the right main pulmonary artery.

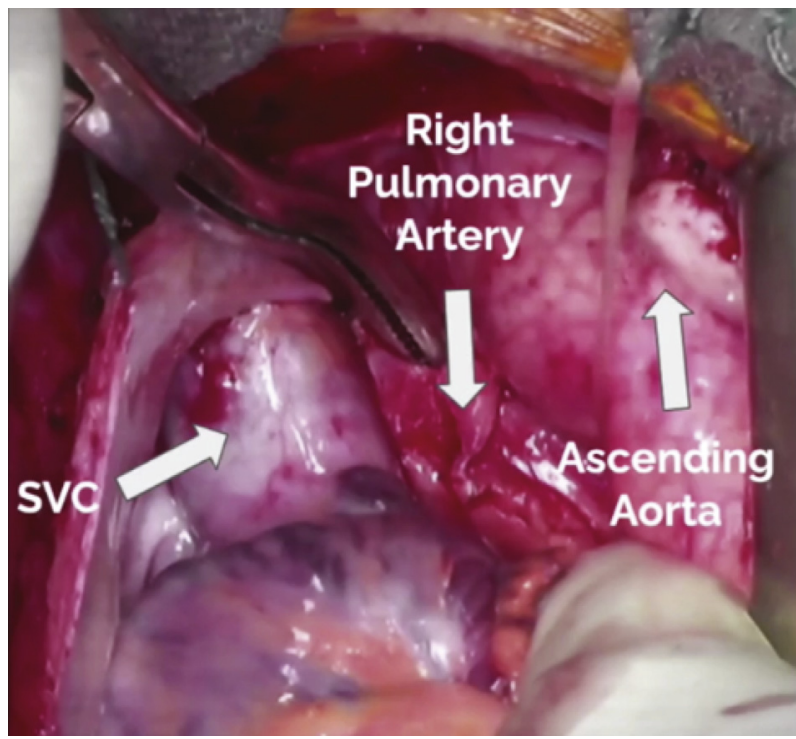

FIGURE E2. Sternotomy view of the right main pulmonary artery between the ascending aorta and superior vena cava $(S V C)$.

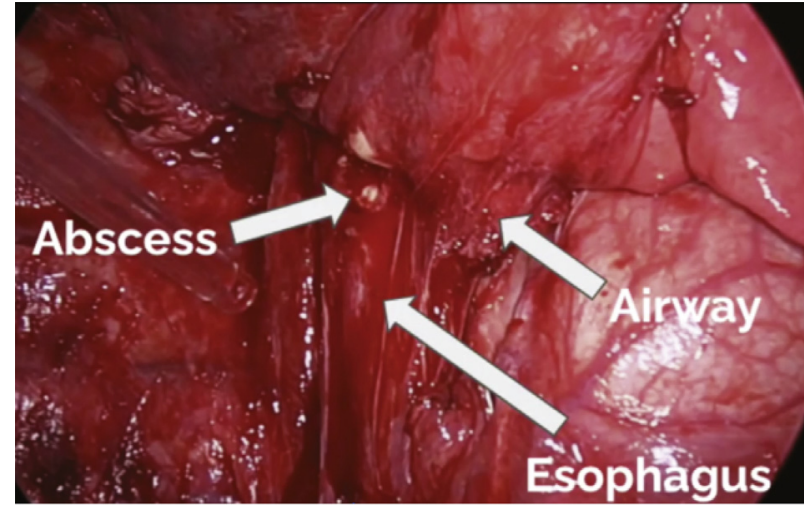

FIGURE E3. Extensive inflammation in the posterior mediastinum. The carina is densely adhered to esophagus. 


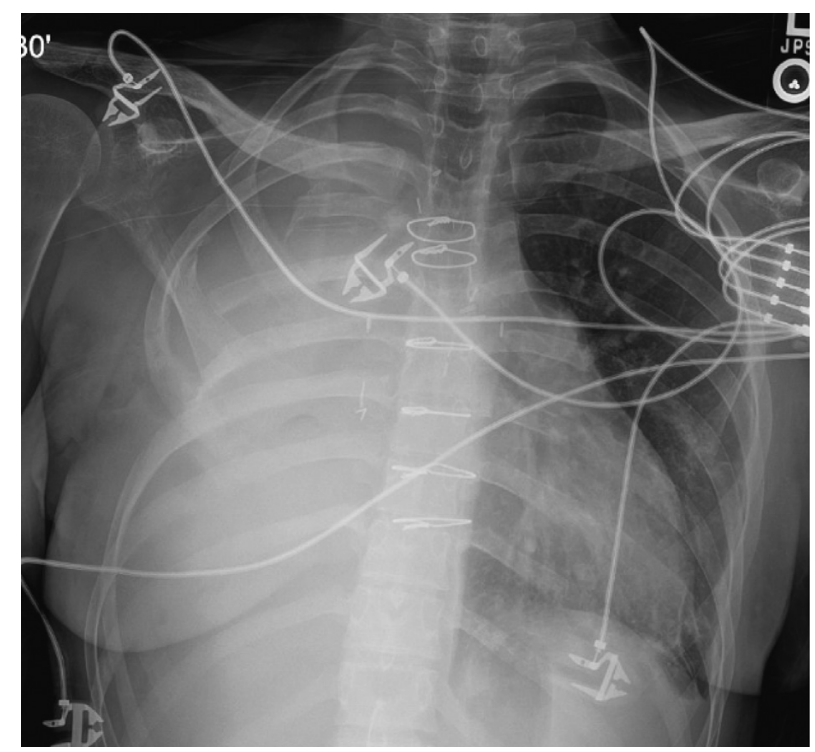

FIGURE E4. One-year follow up chest radiograph after Eloesser flap closure.
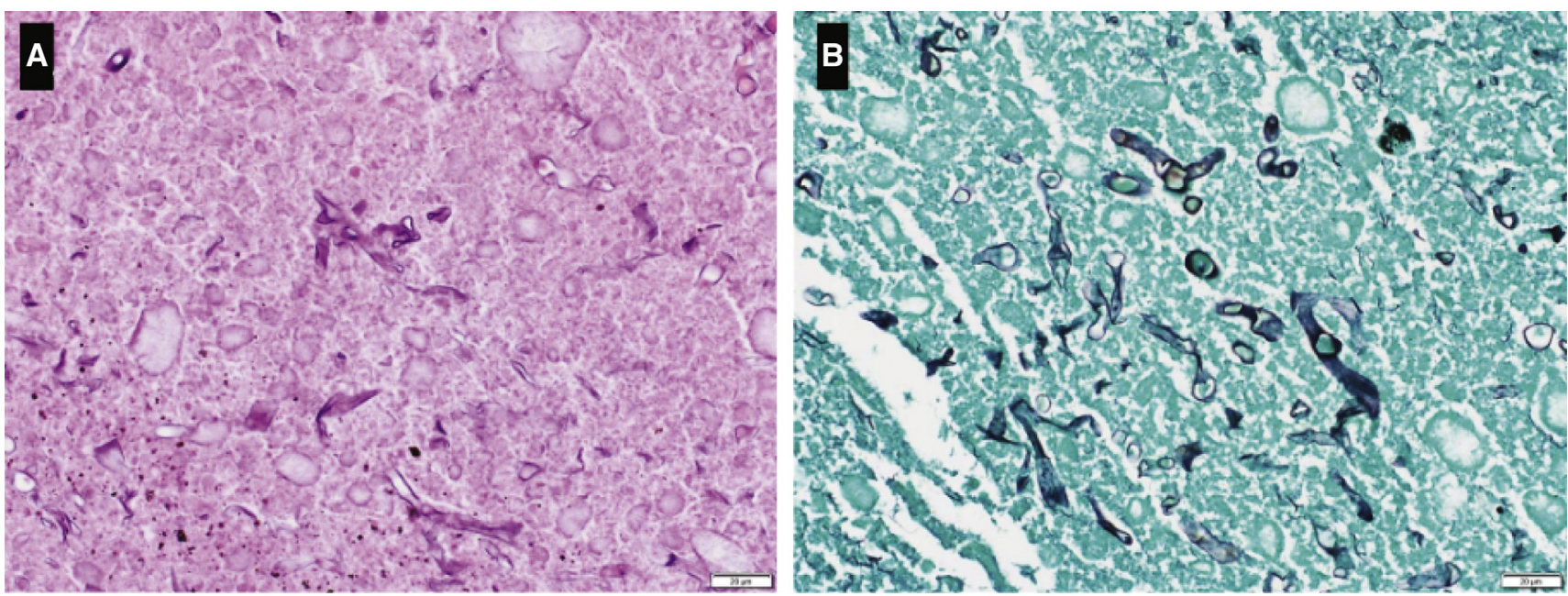

FIGURE E5. Pathology specimen. Hematoxylin and eosin stain (A), and Grocott methenamine silver stain (B). High magnification (200×) examination of necrotic areas of the lung reveals presence of wide-angle nonseptate fungal hyphae with ribbon-like appearance, characteristic of mucormycosis. 